\title{
Application of Al technology in interior design
}

\author{
Zixuan Chen ${ }^{1}$, Xiang Wang ${ }^{2}$ \\ ${ }^{1}$ Department of Architecture and Art, of North China University of Technology, Beijing, Beijing, 10000, China \\ ${ }^{2}$ Department of Architecture and Art, of North China University of Technology, Beijing, Beijing, 10000, China
}

\begin{abstract}
AI technology is coming into our life in recent years. Science and technology also changing and improving our interior design. The overall national strength has grown remarkably, and the level of science and technology has reached the world-class level. This paper starts from the combination of AI and home design, multi-angle analysis of the current development and future prospects. According to the bedroom, kitchen, children's items storage problems to provide solutions. Storage is an important problem for designers, and also a difficult problem for users in their life. However, with the help of AI, it can bring first-class experience for users. This paper will carry out analysis and research from this point.
\end{abstract}

\section{Introduction}

\subsection{Background}

Since the development of AI (Artificial Intelligence). the country has launched relevant policies since 2015 . It has entered the national strategic position since 2016, the plan identifies six specific areas to support AI development, including funding, system standardization, intellectual property protection and international cooperation etc. Internationally, countries' support for the development of AI is not only reflected in the strategy itself, but also reflect on other fields such as economy, society and industry. In December 2018, the European commission and its member states released the AI coordination plan with the theme of "AI made in Europe". It can be seen that the government is determined to raise AI to the national level. It also become an important driving force to lead the development of science and technology.

\subsection{Al and Smart Home Design}

It is a new technical science that extending human intelligence which can studies theories, methods, technologies and application systems for simulating. Interior design with the increasingly close combination of $\mathrm{AI}$ is the inevitable trend of the Internet age. Now the smart home has entered a part of the family. Not only comfort the sense of use, but also make the safety and portability improved to a higher level. Even from an environmental perspective, it can provide a green and sustainable residential space for us.

\subsection{Current Developing Situation}

Smart home has appeared in China for a period of time, mainly reflected in two aspects. There are two kinds of smart home, one is simple intelligence; this kind of consumer is high income people. These products mainly are to upgrade function, such as induction lighting, constant temperature and humidity, etc. Another kind is various brands, such as Haier, Midea, Xiaomi and other brands, have launched a series of collaborative control and work of devices that can be operated by mobile terminals. These products have price advantage, evaluation and market feedback are very good in recent years. But they make the product research and development only in their own brand internal collaboration. But from the overall development of smart home, this rail is too one-sided. Different brand and operation are not compatible, it really has certain limitations from long-term development direction.

\section{Research on the technology of smart home}

\subsection{Demand for smart home apps}

Nowadays, people's life is 'Live can't without mobile phone', this is people's favorite way in the Internet era. Use APP associated with family facilities has been used very widely. APP and intelligent household interaction, provides first-class products, rich experience of goods and services to users. Smartphone is small and portable, became the most popular to use for the control terminal. Relevant professionals believe that the application of APP is a very important platform for Internet technology, and it has contributed to the power of enterprises to agglutinate users. I'm going to write the following part 
based on the mind map of technology of smart home (Figure 1).

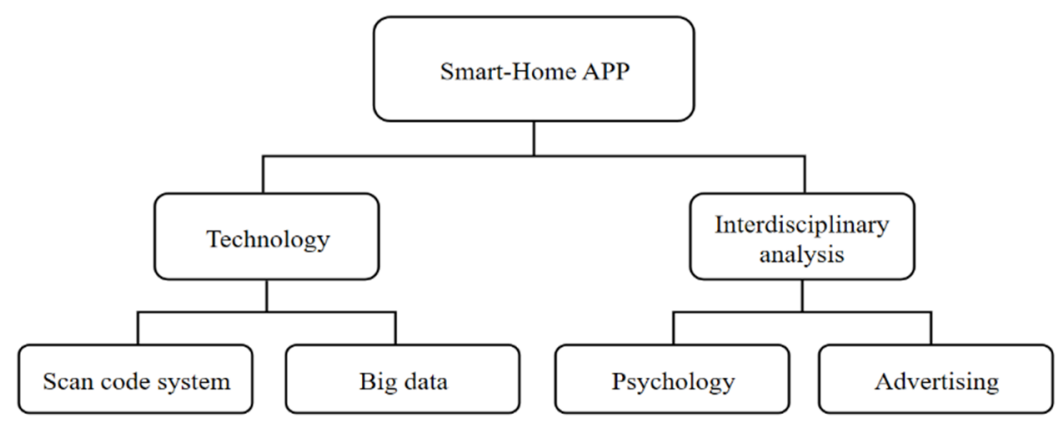

Figure 1. Mind-map of technology of smart home

\subsection{Scan QR code}

At present, there are mainly two types of code recognition technology in the market, fixed and mobile. The main trend of the public is mobile at present, so I choose to use mobile code recognition technology in this system. After scanning the specified device, the information of the QR code can be obtained, such as the material, color, size and so on. As long as there is a network, the decoding process can be compared to the database, read decoding data.

\subsubsection{Big data}

Configure the Hadoop environment and manage it with clouder manager. In order to achieve the technical level of data collection and preprocessing, data storage and management, data processing and analysis, data privacy and security. We extract signals from the data, build a model of users' purchase intention, and focus on the analysis of users' brand preference, product demand, purchase concept, consumption capacity, etc.

\subsection{Interdisciplinary analysis}

\subsubsection{Crossover method}

This is a comprehensive multi-disciplinary research. If you want more comprehensive analysis, it is a method to verify the rationality of the research based on the comprehensive knowledge of multiple disciplines.

\subsubsection{Psychology model building}

To satisfy user experience, interior design needs to meet people's psychological needs, in which psychology plays a leading role. Environmental psychology discusses the environment with the psychology of human beings, starting from the psychological characteristics of human beings and the relationship between human beings and the environment.
It will be a gradual process for new smart products to come into life and be accepted by the public. From the perspective of the designer, Smart-home products as an emerging product, need to give people times to accept. People's psychology will reject the complex appearance of things, and they are more willing to accept seemingly simple things. Therefore, this inspires the designer, if the appearance is seemed easy will make the combination of $\mathrm{AI}$ and household design is more easily accepted by people.

\subsubsection{Combined with the communication value of advertising}

In today's Pan media environment, the definition of media has been greatly expanded. New Internet social media, on the basis of traditional advertising, devote more energy to the process from arousing desire to purchasing action. so that users can complete all five stages of the theoretical model of consumer behavior in one terminal, and minimize the resistance and uncertainty encountered in the process from arousing desire to purchasing action, so as to achieve a relatively ideal conversion rate. Intelligent storage system is a new media terminal integration device with great potential.

\section{Design and analysis of smart-home Storage System}

\subsection{Introduction}

The whole design project is based on the specific storage situation of different places in the family. Project combine with AI focus on people's behavior, storage content, storage partition, suitable for different family conditions. Based on my research results, I formed different emphases according to different people and scenes, and formed a plan to integrate with AI.

\subsubsection{Bright S-Bedroom}

Smart wardrobe needs to provide a clear display of existing clothes. An intelligent interactive system is added in an ordinary wardrobe, assist user's selection and search. Each set of plans is based on own cabinet body, 
analysis can store the volume, improve the utilization rate of the space. The point is according to user's habit made personal customization, make the bedroom clear and better. We need some simple steps to better manage

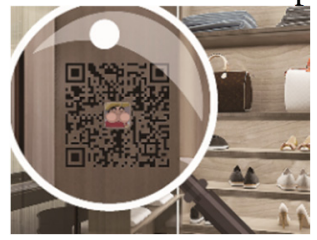

Figure 2.Scan identification closet

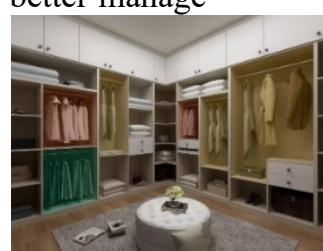

Figure 3.Partition arranges our storage space (Figure 2-5), the following will show the flow chart about the main functions (Figure 6) and additional functions (Figure 7) in bedroom.

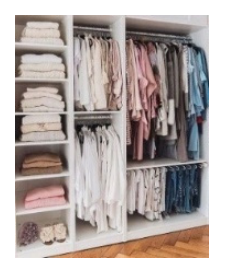

Figure 4.by color

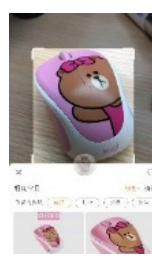

Figure 5.Photo recognition

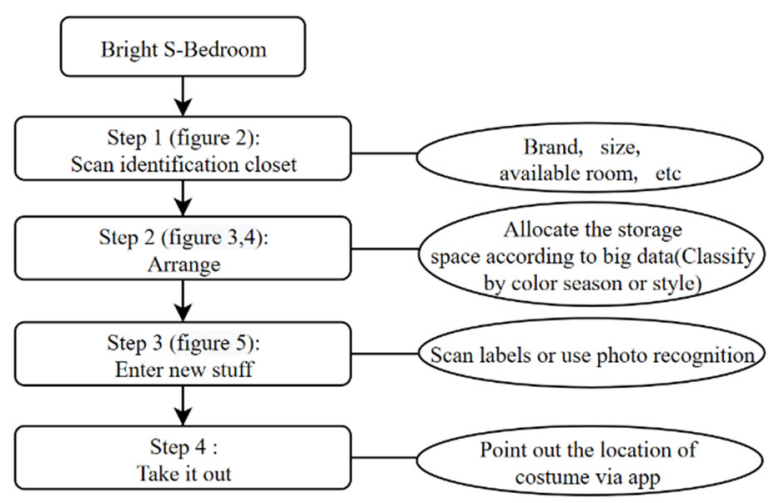

Figure 6. Bedroom system flow chart

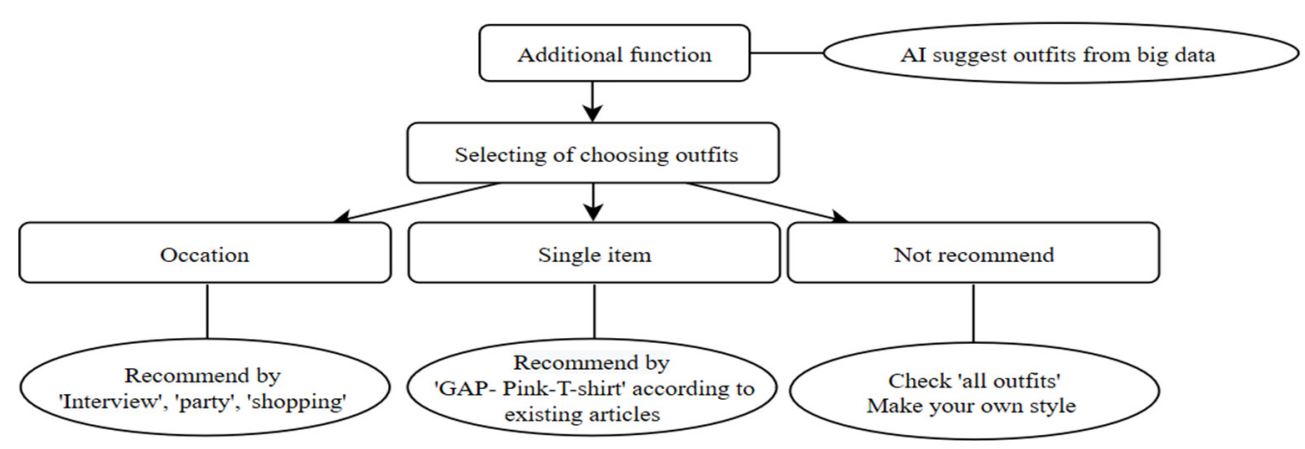

Figure 7. Bedroom additional function flow chart

\subsection{Efficient S-Kitchen}

In most homes, the functionality of the kitchen is especially important. The key word for a good kitchen is "Efficient". Only combine function, appearance and technology can make cooking life better.

But what will be truly make a revolution is technology brings, keep pace with the modern lifestyles while maintaining a high-class aesthetic at the same time.

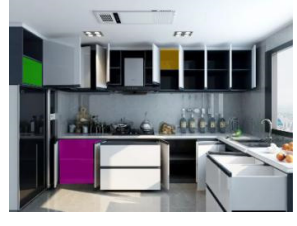

Figure 8.Arrange kitchen

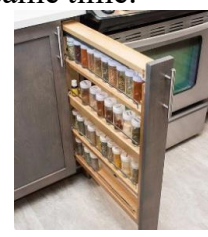

Figure 9. seasoning pot
Smart-Kitchen technology developed in the past 10 years, Solve the storage problem of the kitchen through customized storage modules with different function. In addition to receive a function planning, combined operation of kitchen appliances is achievable. We need some simple steps to better manage our kitchen articles (Figure 8-10), the following will show the flow chart about the main functions (Figure 11) and additional functions (Figure 12) in kitchen.

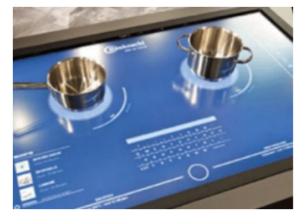

Figure 10 Smart cooker 


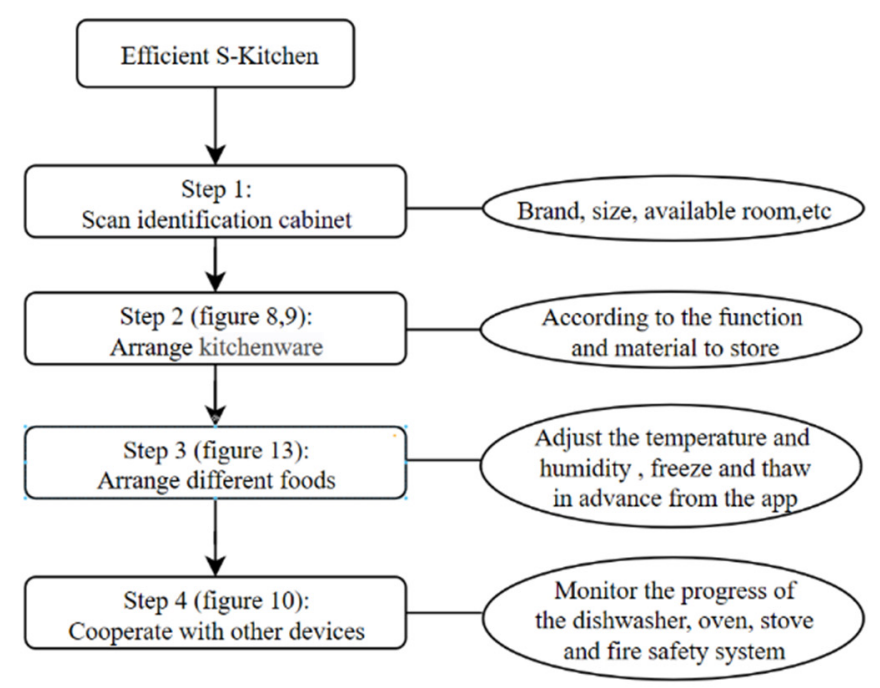

Figure 11. Kitchen system flow chart

This is a refrigerator temperature adjustment device of smart kitchen system designed by Kin team (figure 13),

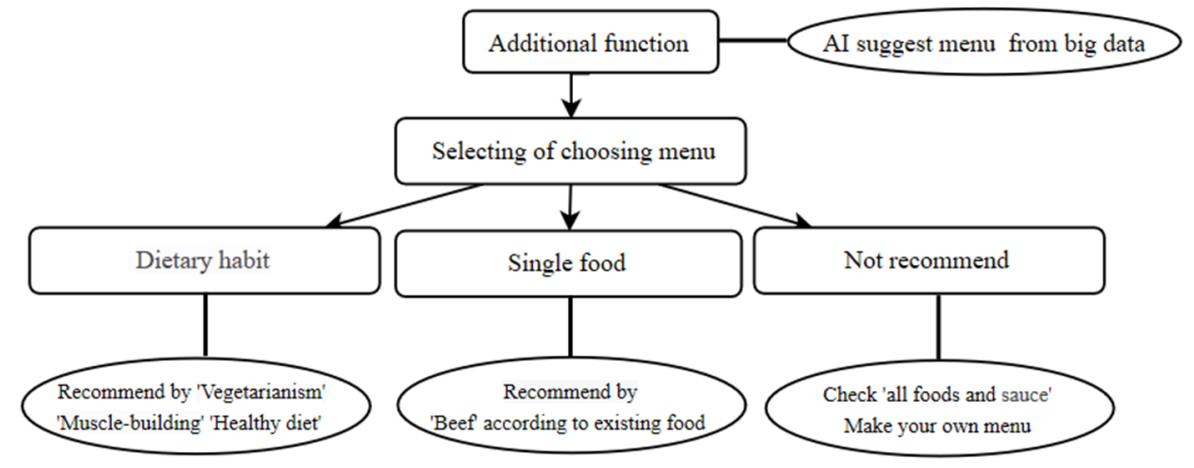

Figure 12. Kitchen additional function flow chart which can adjust the temperature according to different regions and can freeze drinks in advance.

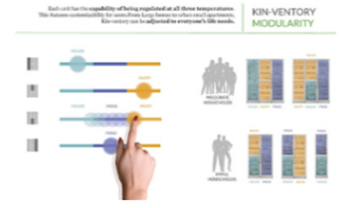

Figure 13. Temperature controller
In addition, a food sharing community can be established. Users can share the food with each other, It not only use for the control terminal of the S-kitchen, but also becomes the perfect carrier to share their daily cooking life.

\section{3. "GROW HAPPINESS" Children system}

This provides a new idea for the storage of children's items. Child always have the action of eating hands, eating toys, so their weak resistance needs more powerful cleaning and degerming system. Based on the central idea of sustainable development, better to maximize the service life of children's product. The theme of this Smart children system is "clean" and "sustainable". The following will show the flow chart about the main functions (Figure 14) in children's room. 


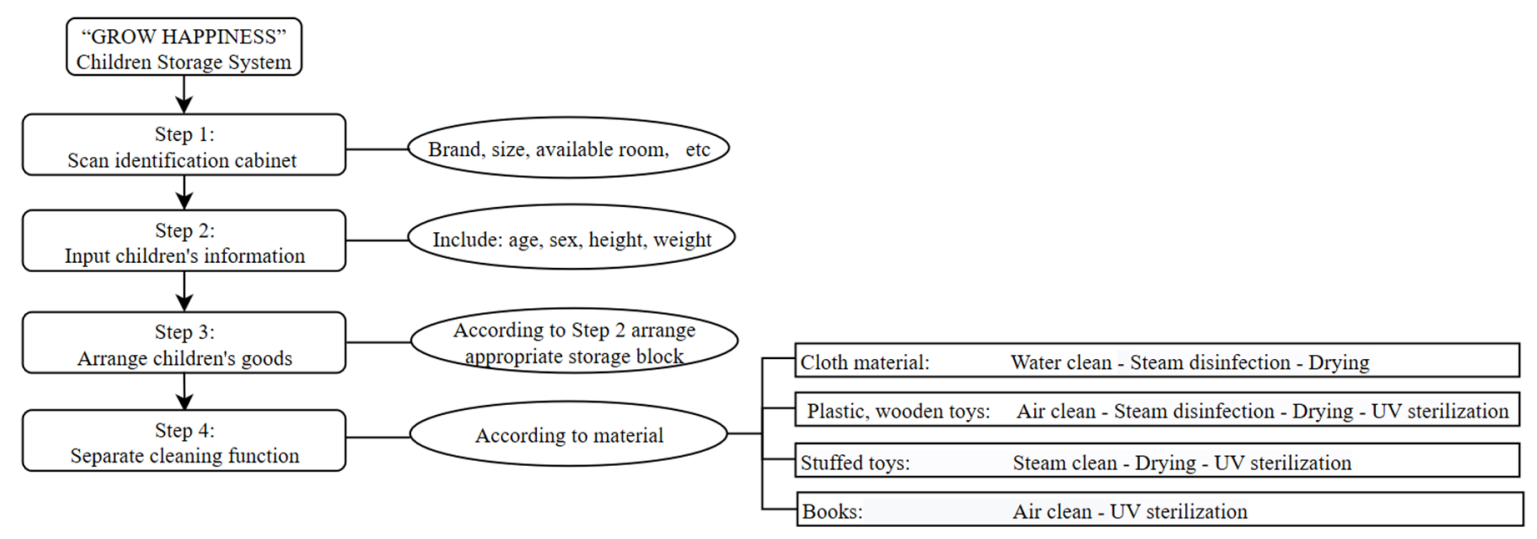

Figure 14. Children system flow chart

The cabinet interior uses movable board, which can be disassembled and combined freely. Whenever the children reach a new stage of growth, the size and quantity of child items change a lot, so the system would put forward new adjust suggestions. For the parents, it also can adjust according to habits of adults (figure 15).
The board cannot use metal nails with difficult operation and hinge operation. The principle of Chinese mortise and tenon joint is an inspiration which can form a convenient and stable segmentation module.

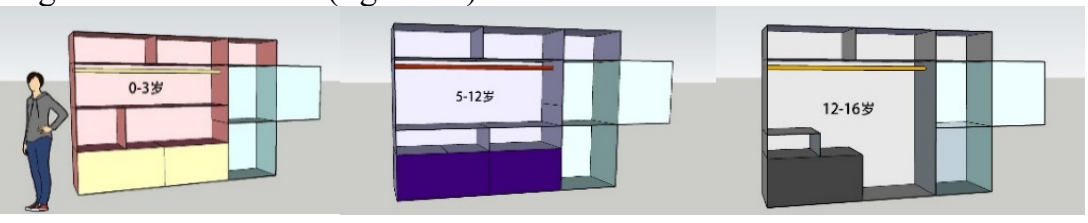

Figure 15. Closet in Different age

\section{Future trends and business value}

Smart home is born in the network era. However, smart home want to promote in the future, will be face many problems and difficulties such as policy support and technological development, which play an important role in the development.

From the perspective of market, intelligent storage system is closely related to all aspects of users' life. When it enters many families, it has the opportunity to collect more real and timely data from users. This makes more and more brands pay attention to big data technology and the precise delivery behind it. These data are valuable wealth whether they are used as chips to exchange resources with other business partner or to assist other products of their own to go online.

For users, comprehensive insight can bring more targeted recommendations. Not only can we find the products we are satisfied with efficiently, but also can we save the time to buy the daily consumables repeatedly. Under the virtuous circle of mutual benefit between consumers and manufacturers, smart wardrobe system is expected to become the next influential bilateral market in the consumer market.

But if want to be widely used, the first priority is to regulate the use. Specifically, to set industry standards, in terms of price and safety issues, to provide security for businesses and users. Another problem is about the longterm use of the system maintenance problems, should be restricted some manufacturers which lack of after-sale protection. In addition, there is a new problem, that is network information security. The protection of user privacy has become a key problem in the information age.

\section{Conclusions}

The idea of this project is following the trend of scientific and technological, which is meet young people's interest. The technology and functional design in the project are basically existing technologies. With the development of technology, it will be upgraded sooner or later, which can make the cost lower and the efficiency higher. The article analysis each respect of intelligent home system, and expand thinking to form this project, provided new possibility in this area.

\section{Acknowledgements}

This paper was funded by the education project of North China University of Technology in 2020, and was one of the phased achievements of Research and Experiment on Innovation and Entrepreneurship Education for Design College Students (215051360020XN156).

\section{References}

1. Xu, Z., Wu, H. (2019) Modular design analysis of intelligent furniture. SP., 12: 128.

2. Li, S. (2019) Study on intelligent furniture in living smart home system. JUSHE., 34: 21+26. 
3. Cai,Y., Xie, X. (2020) Research on modern interior design under the influence of environmental psychology. JUSHE., 04: 16.

4. Deng, Q., Jin, D. (2019) Research on Smart Home System -- Taking wardrobe storage as an example. Home Drama., 17: 209-210.

5. Chen, Y., Zhou, S., Ai, J., (2016) Design and research of intelligent furniture based on user experience. https://www.cnki.net/.

6. Tao, J., Zhang, J. (2019) Research on kitchen storage space design based on storage behavior. https://www.cnki.net/.

7. Lu, H., Wu, G. (2014) Research on humanized design of storage system in home space. https://www.cnki.net/.

8. Liu, Y., Feng, M. (2015) Study on the influence of user's habits on the design of household products. https://www.cnki.net/. 